\title{
A REALIZATION OF THE ADDITIVE WITT GROUP
}

\author{
KENNETH NEWMAN ${ }^{1}$ AND MOSS E. SWEEDLER ${ }^{2}$
}

\begin{abstract}
For each prime $p$ we construct in several ways a canonical sequence of divided powers and give an elementary proof that its coefficients lie $\mathbf{Z}_{(p)}$.
\end{abstract}

Introduction. Let $H$ be a commutative, cocommutative irreducible Hopf algebra over $\mathbf{Q}$, the rationals. A sequence of elements in $H,{ }^{0} x=1,{ }^{1} x$, ${ }^{2} x, \ldots$, is called a sequence of divided powers (SDP) if, for all $n, \Delta^{n} x=$ $\sum_{i=0}^{n}{ }^{i} x \otimes{ }^{n-i} x$. In this paper we consider SDP's such that for a fixed prime $p$, each $p^{n}$ th divided power is algebraically independent of the previous terms in the sequence, while the other divided powers are algebraically dependent on the previous terms. Using the exponential Taylor series we give several realizations of this sequence (including a rather complicated formula) and also give an elementary proof that the coefficients of the polynomials representing the elements in the sequence lie in $\mathbf{Z}_{(p)}$. (This was previously shown in [3].)

For convenience, we will call a power series in $T, 1+\sum_{i=1}^{\infty} x_{i} T^{i}$, an SDP if $1, x_{1}, x_{2}, \ldots$ is an SDP.

Also we will denote the primitives of $H, P(H)$.

Lemma 1 [1, Proposition 2]. The formal power series in $T, \exp \left(\sum_{i=1}^{\infty} x_{i} T^{i}\right)$ is an SDP if and only if each $x_{i}$ is primitive.

Corollary 2 [1, Corollary 3]. $1+\sum x_{i} T^{i}$ is an SDP if and only if the coefficient of $T^{i}$ in $\log \left(1+\sum_{i=1}^{\infty} x_{i} T^{i}\right)$ is a primitive for all $i$.

Lemma 3 [1, Theorem 4]. Give $H_{n}=\mathbf{Q}\left[X_{1}, X_{2}, \ldots, X_{n}\right]$ a graded Hopf algebra structure by defining $\Delta X_{i}$ so that $1, X_{1}, X_{2}, \ldots, X_{n}$ is an SDP and by letting $\operatorname{deg} X_{i}=i$. Then there exists a homogeneous extension to $1, X_{1}, X_{2}, \ldots, X_{n}$. We denote such an extension $P_{n+1}$.

Lemma 4. Assume that $J=\mathbf{Q}\left[X_{1}, X_{2}, \ldots, X_{m}\right]$ is a graded irreducible Hopf algebra and contains an $S D P 1,{ }^{1} x,{ }^{2} x, \ldots,{ }^{n} x$ such that

(1) ${ }^{i} x$ is homogeneous of degree $i$ for all $i$;

Received by the editors June 13, 1978.

AMS (MOS) subject classifications (1970). Primary 16A24.

Key words and phrases. Sequence of divided power, Witt vectors, Artin-Hasse exponential.

'This research was partially supported by National Science Foundation Grant no. NSF 76-066 38A01.

${ }^{2}$ This research was partially supported by National Science Foundation Grant no. NSF MCS 73-04876. 
(2) ${ }^{\mathrm{l}} x=X_{1}$;

(3) for $2 \leqslant i \leqslant m, \exists k_{i} \geqslant 2$ such that $X_{i}={ }^{k_{i}} x$ and $i<j \Rightarrow k_{i}<k_{j}$.

Then there exists a unique homogeneous extension ${ }^{n+1} x$ to $1,{ }^{1} x$, ${ }^{2} x, \ldots,{ }^{n} x$.

Proof. Existence. By Lemma $3, H_{n}$ contains a homogeneous extension $P_{n+1}$ to $1, X_{1}, X_{2}, \ldots, X_{n}$. Now $\varphi: H_{n} \rightarrow J$ via $X_{i} \rightarrow{ }^{i} x$ will be a graded Hopf algebra map and $\varphi\left(P_{n+1}\right)$ will be the desired extension.

Uniqueness. A second homogeneous extension would differ from the first by a homogeneous primitive $d$ of degree $n+1$. We show $d=0$ as follows: by [4, Theorem 13.0.1, p. 274] over a field of characteristic 0 any cocommutative irreducible Hopf algebra is the universal enveloping algebra of the Lie algebra of primitives and, since $J$ is also commutative, $J$ is generated by any linear basis of $P(J)$ as a polynomial ring, i.e., if $\left\{d_{\alpha}\right\}$ is a basis for $P(J)$ then $\left\{d_{\alpha}\right\}$ is an algebraically independent generating set in $J$. If $0 \neq d$, then it may be included in a basis for $P(J)$ and hence in an algebraically independent generating set of $J$. On the other hand, an element may be included in an algebraically independent generating set only if it is of the form:

$$
\gamma+\sum_{i=1}^{m} \lambda_{i} X_{i}+R\left(X_{1}, X_{2}, \ldots, X_{m}\right)
$$

with $\gamma, \lambda_{1}, \ldots, \lambda_{m} \in \mathbf{Q}$, at least one $\lambda_{i}$ not zero, and $R$ in the square of the ideal generated by $X_{1}, X_{2}, \ldots, X_{m}$. But an element of the form ( $\dagger$ ) cannot be homogeneous of degree greater than $n$ since $X_{i}=k^{i} X$ is homogeneous of degree $k_{i}$. Thus $d$ which is homogeneous of degree $n+1$ cannot be written in form $(\dagger)$ and must therefore be zero. Q.E.D.

Note that this lemma implies that the extension in Lemma 3 is unique.

We will be especially interested in the situation of the above lemma where for a prime $p, X_{i}={ }^{p}{ }_{x}^{x}$, for all $i \geqslant 0$. (For convenience we will start subscripting the $X$ 's at 0 .) Using the above lemma and induction we know that $\exists$ ! homogeneous SDP: $1, P_{1, p}, P_{2, p}, \ldots$ in $\mathbf{Q}\left[X_{0}, X_{1}, X_{2}, \ldots\right]$ such that $P_{p^{i}, p}=$ $X_{i}$ and $\operatorname{deg} X_{i}=p^{i}$.

Another method of constructing this sequence and one leading to a computational realization derives from Lemma 1. Consider

$$
\exp \left(X_{0} T+\left(X_{1}-Q_{1}\right) T^{p}+\left(X_{2}-Q_{2}\right) T^{p^{2}}+\ldots\right)
$$

where $Q_{i}$ is the coefficient of $T^{p^{i}}$ in

$$
\exp \left(X_{0} T+\left(X_{1}-Q_{1}\right) T^{p}+\cdots+\left(X_{i-1}-Q_{i-1}\right) T^{p^{i-1}}\right) .
$$

Note that the coefficient of $T^{p^{\prime}}$ in (*) is $X_{i}$. Further if we inductively define

$$
\Delta X_{i}=\Delta Q_{i}+1 \otimes\left(X_{i}-Q_{i}\right)+\left(X_{i}-Q_{i}\right) \otimes 1,
$$

we have that $X_{0}$ and $X_{i}-Q_{i}$ are primitive for all $i$. Thus (*) is an SDP. The uniqueness part of Lemma 4 ensures that this is the same SDP defined above. 
A somewhat more "magical" algorithm using the same idea can be stated as follows:

Consider $\exp \left(X_{0} T\right)$ and replace the coefficient of $T^{p}$ by $X_{1}$. Take log and discard all $T^{i}$ with $i>p$. Then all coefficients are primitives and exp of this will be an SDP. Now replace the coefficient of $T^{p^{2}}$ with $X_{2}$, take $\log$, discard $T^{i}$ with $i>p^{2}$, etc.

In any case, we see from $(*)$ that if we inductively define

$$
Q_{i}=\sum_{m_{0}+p m_{1}+\cdots+p^{i-1} m_{i-1}=p^{i}} \frac{X_{0}^{m_{0}}}{m_{0} !} \frac{\left(X_{1}-Q_{1}\right)^{m_{1}}}{m_{1} !} \cdots \frac{\left(X_{i-1}-Q_{i-1}\right)^{m_{i-1}}}{\left(m_{i-1}\right) !}
$$

then

$$
P_{n, p}=\sum_{m_{0}+p m_{1}+\cdots+p^{\prime} m_{t}=n} \frac{X_{0}^{m_{0}}}{m_{0} !} \frac{\left(X_{1}-Q_{1}\right)^{m_{1}}}{m_{1} !} \cdots \frac{\left(X_{t}-Q_{t}\right)^{m_{t}}}{m_{t} !}
$$

where $p^{t}$ is the largest power of $p$ less than or equal to $n$.

For example if $n<p$ then $P_{n, p}=x_{0}^{n} / n$ !. If $p<n<p^{2}$ then

$$
P_{n, p}=\sum_{m_{0}+p m_{1}=n} \frac{X_{0}^{m_{0}}}{m_{0} !} \frac{\left(X_{1}-X_{0}^{p} / p !\right)^{m_{1}}}{m_{1} !}
$$

since $Q_{1}=x_{0}^{p} / p !$.

What is remarkable about this sequence is that the coefficients lie in $\mathbf{Z}_{(p)}$, i.e., the denominators are relatively prime to $p$ and therefore one can use the $P_{n, p}$ to construct SDP's over fields of char $p$. We give an elementary proof using Witt vectors and the Artin-Hasse exponential.

In $\mathrm{Q}\left[Y_{0}, Y_{1}, \ldots\right]$ let $\phi_{n}=Y_{0}^{p^{n}}+p Y_{1}^{p^{n-1}}+\cdots+p^{n} Y_{n}$. If we let each $\phi_{n}$ be primitive then the $\Delta Y_{i}$ are defined inductively.

Lemma 5 [2, Chapter $\left.\mathrm{V}, \S 4, \mathrm{no}^{\circ} 4.1, \mathrm{p} .617\right]$. Let the coefficient of $T^{i}$ in

$$
\exp \left(-\phi_{0} T-\phi_{1} \frac{T^{p}}{p}-\phi_{2} \frac{T^{p^{2}}}{p^{2}}-\ldots\right)
$$

be $E_{i}$. Then, $E_{i} \in \mathbf{Z}_{(p)}\left[Y_{0}, Y_{1}, \ldots\right]$ for all $i$.

Now since $\phi_{0}, \phi_{1}, \ldots$ are primitives $(* *)$ is an SDP with coefficients in $\mathbf{Z}_{(p)}$. Further, since $E_{p^{n}}=Y_{n}+$ (polynomial in $\left.Y_{0}, Y_{1}, \ldots, Y_{n-1}\right)$, the coefficients of the $T^{i}$ can be expressed in terms of the $E_{p^{n}}$. As the substitution process takes place over $\mathbf{Z}_{(p)}$, we have just shown that there is an SDP with coefficients in $\mathbf{Z}_{(p)}\left[E_{1}, E_{p}, E_{p^{2}}, \ldots\right]$ such that the $p^{n}$ th term is $E_{p^{n}}$. Using the uniqueness part of Lemma 4 we have (with $X_{i}$ replacing $E_{p^{i}}$ ):

THEOREM 6. The coefficients of $P_{n, p}$ lie in $\mathbf{Z}_{(p)}$ for all $n$. 


\section{BIBLIOGRAPHY}

1. A. Berkson and K. Newman, Constructing sequences of divided powers. II, Proc. Amer. Math. Soc. 72 (1978), 11-15.

2. M. Demazure and P. Gabriel, Groupes algébriques, Tome 1, North-Holland, Amsterdam, 1970.

3. K. Newman, Constructing sequences of divided powers, Proc. Amer. Math. Soc. 31 (1972), 32-38.

4. M. E. Sweedler, Hopf algebras, Benjamin, New York, 1969.

Department of Mathematics, University of Illinois at Chicago Circle, Chicago, ILLINOIS 60680

Department of Mathematics, University of Massachusetts, Boston, Massachusetts 02125 (Current address of Kenneth Newman)

Department of Mathematics, Cornell University, Ithaca, New York 14853 (Current address of M. E. Sweedler) 